\title{
MINDFULNESS E INTELIGENCIA EMOCIONAL EN EL AFRONTAMIENTO DE CONFLICTOS INTRAPERSONALES E INTERPERSONALES
}

\author{
Rosa Delia Martín Rodríguez \\ Universidad Internacional de la Rioja (UNIR) \\ rosa.martin@unir.net \\ Isabel Luján Henríquez \\ Universidad de Las Palmas de Gran Canaria (ULPGC) \\ isabel.lujan@ulpgc.es
}

Recepción Artículo: 16 mayo 2021 Admisión Evaluación: 16 mayo 2021 Informe Evaluador 1: 22 mayo 2021 Informe Evaluador 2: 25 mayo 2021 Aprobación Publicación: 01 junio 2021

\section{RESUMEN}

Este trabajo tiene como objetivo fundamental analizar la relación entre las competencias y habilidades emocionales y el mindfulness -como toma de conciencia de atención plena- y como recursos y estrategias eficaces para la gestión y resolución de conflictos intrapersonales e interpersonales. Para ello, se llevó a cabo una investigación cuasi-experimental, de tipo tanto cuantitativo como cualitativo en la que intervinieron 117 estudiantes universitarios sin ningún tipo de patología y que habían participado en un programa de entrenamiento en mindfulness. Los resultados nos indican que las variables personales de tipo emocional y la toma de conciencia plena mantienen una alta relación y subyacen en un afrontamiento adecuado de los conflictos por lo que contribuyen al bienestar personal y social. Esto nos permite reflexionar sobre la importancia del conocimiento de estas variables por parte de los/as profesionales en la resolución de conflictos, para que en su actuación negociadora 0 mediadora faciliten a las personas en el proceso no solo resolver el conflicto de forma satisfactoria y de llegar a un acuerdo, sino la toma de conciencia de su transformación personal.

Palabras clave: mindfulness; toma de conciencia plena; gestión de conflictos; resolución de conflictos

\section{ABSTRACT}

Mindfulness and emotional intelligence in problem-solving within and among individuals. The main objective of this paper is to analyse the link between emotional competences and skills, and mindfulness. This includes full attention awareness and efficient resources and strategies to manage conflicts and solve problems within and among individuals. To do so, both quantitative and qualitative quasi experimental research was conducted. 117 university students with no disorders who had taken a training course in mindfulness participated in the experiment. Results show that personal variables related to emotions and full awareness are closely 
intertwined. Being at the basis of appropriate management of conflict, they are key to personal and social wellbeing. This makes us realise how important it is for problem-solving professionals to know about these variables. By taking them into account in mediation and negotiation processes, they will be able to help people solve their conflicts and reach agreements successfully while becoming aware of their personal transformation.

Keywords: Mindfulness; full awareness; conflict management; problem-solving

\section{INTRODUCCIÓN}

Actualmente es objeto de estudio de gran interés la profundización en el conocimiento sobre los beneficios de la práctica del mindfulness para el desarrollo de las habilidades de autocontrol y gestión emocional, relacionados con la toma de decisiones asertivas con la finalidad de percibir y conseguir desenvolvernos de la forma más óptima posible en el medio en el que vivimos.

La mejora de las relaciones interpersonales, con lo que conlleva como sentimiento de bienestar subjetivo en las personas, procede de cuando son capaces de reflexionar sobre sus actos sin emitir juicios de valor sobre la experiencia vivida. Según Siegel, (2010, 2012) y Kabat-Zinn (2012), las personas que muestran mayor nivel competencial en habilidades de conciencia plena o mindfulness, centradas en el presente de forma consciente y con un mayor equilibrio emocional, aportan respuestas más asertivas en el ambiente en el que se desenvuelven. Es decir, que la persona siente una mayor sensación de bienestar personal en relación a las relaciones que mantiene con los demás y en cómo vive su vida cuando es capaz de desarrollar y mantener una atención especial a la experiencia presente en el momento actual aceptándola sin juicios de valor y libre de control.

Simón (2015) señala que la toma de conciencia plena, a través de la observación interna de la persona es el proceso que permite identificar la emoción negativa subyacente y, en consecuencia, regular la respuesta. Por su parte, Redorta (2021) resalta que "la toma de conciencia respecto de algo supone la capacidad de medir las consecuencias de lo que está sucediendo" (p. 57)

Partimos de la base de la importancia que supone la incidencia de la relajación para la activación cognitiva. Su práctica transforma los paradigmas mentales en conductas personales de nuevos modelos de vida y en hábitos de bienestar que disminuyen las afecciones físicas provocadas por estrés, depresión o ansiedad generando salud mental. Asimismo, y desde una visión cognitivo-conductual consideramos, al igual que Hernández-Guanir (2002 y 2010) que cuando una persona se muestra ante la realidad, siendo el protagonista principal, adquiere un modo especial de enfocar, de reaccionar o de interpretar esa realidad en diferentes situaciones.

Por otro lado, y dentro de nuestro interés de investigación sobre la importancia de la inteligencia emocional en la solución de conflictos, la teoría de inteligencias múltiples aportada por Gardner (2001) nos habla, entre otras, de dos tipos de inteligencia: la inteligencia interpersonal, y la inteligencia intrapersonal o satisfacción interna que sentimos cuando nuestra vida se halla en armonía con nuestros sentimientos y cuando somos capaces de tener un mayor autoconocimiento y de analizar nuestros pensamientos, sentimientos para conocer qué nos sucede para actuar de forma adecuada.

Con frecuencia, se recomienda la práctica de la atención plena cuando las personas están bajo situaciones de alto estrés 0 ansiedad, enfermedades crónicas, circunstancias personales críticas, conflictos intrapersonales y conflictos relacionales en entornos próximos, etc. Sin embargo, en muy contadas ocasiones se sugiere su práctica en personas sanas. Es decir, hasta ahora la práctica de la atención plena mayoritariamente se ha recomendado como terapia reparadora, no como potenciación de las habilidades socioemocionales de personas sanas. Pero desde nuestro planteamiento, desde la perspectiva que nos aporta la psicología positiva (Seligman, 2011; Seligman y Cskszentmihalyi, 2000; Siegel, 2010), consideramos que no solo nos debemos centrar en las personas con dificultades, sino, además, en luchar por la optimización del desarrollo de las personas sanas desde un compromiso de potenciación de sus capacidades y habilidades para un afrontamiento adecuado de las distintas situaciones y para resolver los conflictos personales y de relaciones interpersonales propios del devenir de la vida.

La importancia de desvelar los elementos que subyacen en los conflictos y en la forma de afrontamiento de los mismos radica en que una adecuada resolución contribuye a la buena convivencia y a vivir en paz, bien con 
uno mismo o bien en sociedad. Es un tema que, tal y como señala Vinyamata (2019, p. 16), en "todas las culturas y épocas, las personas han procurado reflexionar sobre las causas, los orígenes y las consecuencias de los conflictos".

Al igual que otros autores (Redorta, 2019), concebimos el conflicto, si se afronta de forma adecuada, como fuente de aprendizaje, además, como oportunidad constructiva y productiva, pues "en el mundo interno y microespacios nos permite ahondar en el autoconocimiento, reflexionar sobre nuestras capacidades y debilidades, descubrir nuestro estilo de afrontamiento, posicionarnos ante los problemas y, en suma, modelar nuestras relaciones" (Luján 2015, p. 19).

En la misma línea que Redorta (2019) consideramos que es necesario descubrir cuáles son las causas que generan los conflictos. La presencia de variables emocionales tales como el optimismo, la flexibilidad, la conexión emocional, y el afrontamiento adaptativo conductual se nos han hecho presentes en otras investigaciones en las que aparecen estas competencias socioemocionales en procesos de negociación en resolución de conflictos (Rodríguez-Trueba, Rodríguez-Mateo y Luján, 2014). Asimismo, distintas variables emocionales cobran especial importancia como competencias en la resolución de conflictos. No en vano, mediadores profesionales con larga experiencia en procesos de mediación señalan la importancia del "reconocimiento de aptitudes y habilidades resilientes en los mediados en el proceso mediador como son el pensamiento alternativo, el optimismo, la actitud frente a la adversidad y la adecuada gestión de las emociones. El ser flexibles y estar más dispuestos al cambio favorece en los mediados la transformación positiva del proceso (Luján y Díaz Calcines, 2018, p. 156).

Creemos que desarrollar estrategias que normalmente son utilizadas para anticipar, interpretar, reaccionar, evaluar y explicar la realidad en distintas situaciones interaccionales afectan a la identidad y son las principales responsables de nuestro bienestar y de la forma de gestionar y afrontar nuestras incertidumbres, además de manejar adecuadamente nuestros conflictos con nosotros mismos y con los demás. Por ello, queremos analizar los efectos 0 beneficios de un programa mindfulness y su relación con las variables emocionales en el afrontamiento de conflictos.

\section{OBJETIVOS}

1. Conocer el nivel de normalidad de la muestra en las variables de inteligencia emocional (Percepción, Comprensión y Regulación emocional) en los participantes en un programa mindfulness.

2. Conocer el estado de atención plena antes y después de participar en un programa mindfulness.

3. Conocer, previa aplicación de un programa mindfulness, la relación entre variables de inteligencia emocional y atención plena.

4. Analizar la importancia que los sujetos participantes atribuyen a la inteligencia emocional y a la atención plena en el afrontamiento de conflictos intrapersonales e interpersonales.

\section{Hipótesis:}

- Existe estrecha relación entre la atención plena y las variables de inteligencia emocional.

-El programa Mindfulness-ProAp (Martín, Luján y Rodríguez-Mateo, 2015, en Martín, 2015) mejora la competencia de "atención plena".

- Las personas que participan en programas mindfulness se sienten más seguras y afrontan de forma más adecuada las situaciones que les generan conflicto bien consigo mismo o con los demás.

\section{PARTICIPANTES}

En esta investigación han participado un total de 117 estudiantes universitarios de Las Palmas de Gran Canaria de los cuales 34 pertenecen al género masculino y 83 al género femenino, cuyas edades estaban comprendidas entre los 18 y los 53 años, siendo la mayor frecuencia entre los 20 y los 23 años, obteniendo una media de 24 años. Cursan estudios en diversas especialidades: Técnico Superior en Gestión de Alojamientos Turísticos, Gestión y Técnico Superior en Administración y Finanzas, Técnico Superior en Educación Infantil y Grado en Magisterio. 
La participación en el programa era voluntaria. La promoción se realizó mediante una campaña de información transmitida por los docentes en el aula, carteles publicitarios y medios online (facebook, whatsApp,...).

\section{METODOLOGÍA}

\section{Diseño}

Se ha utilizado metodología de investigación cuantitativa y cualitativa. En la investigación cuantitativa, descriptiva, correlacional y cuasi-experimental se analiza, por un lado, la presencia de las habilidades emocionales de percepción, comprensión y regulación emocional en los participantes en la investigación, y, por otro, la influencia de estas habilidades en la competencia de atención plena. Es metodología cuasi-experimental porque no se manipulan experimentalmente las variables que se van a tomar en consideración.

Por su parte, en la investigación cualitativa, en situaciones de focus group, se recogían, en una puesta en común, las valoraciones de la importancia que los participantes, como informantes clave por su experiencia obtenida durante el entrenamiento en mindfulness, atribuían a la toma de conciencia plena y a las habilidades y competencias emocionales en la gestión de conflictos consigo mismos y con los demás.

\section{Variables}

- Predictoras: Variables de naturaleza cognitivo-emocional; Habilidades de inteligencia emocional: percepción, comprensión y regulación emocional; Programa de intervención Mindfulness-ProAp.

- Criterio: Mayor o menor atención plena después de haber participado en el programa Mindfulness-ProAp.

\section{Instrumentos}

-Instrumento diseñado ad hoc de datos sociodemográficos que contempla los siguientes datos: género, edad y nivel de estudios universitarios.

-Programa Mindfulness-ProAp (Martín, Luján y Rodríguez-Mateo, 2015) para la mejora de atención plena, basado en MBSR (Mindfulness - Based Stress Reduction- MBSR, Kabat-Zinn, 2003).

- Escala de inteligencia emocional TMMS-24 basada en Trait Meta-MoodScale (TMMS) del grupo de investigación de Salovey y Mayer y que ha sido adaptada al castellano por Fernández-Berrocal, Extremera y Ramos (2004). Esta escala cumple los requisitos de fiabilidad necesarios para ser utilizada como instrumento básico en la investigación (0.80). Aborda los factores de percepción, comprensión y regulación emocional. Está constituida de 24 enunciados cortos a los que se debe responder en una escala graduada Likert con 5 alternativas de respuesta.

- Test de Mindfulness o Atención Plena (MAAS). León, Fernández, Grijalvo , y Nuñez, (2012) e Inchausti, Prieto y Delgado. (2013) corroboran la eficacia y validez del autoinforme con una Cronbach de 0.89. Por tanto, el Test MASS es un instrumento fiable y válido, por lo que se consideró adecuado su empleo para medir la atención plena de los participantes en esta investigación.

-Narrativas experienciales, en sesiones de focus group, de participación en el programa Mindfulness-ProAp.

\section{Procedimiento}

La investigación se realizó en tres fases:

Fase preparatoria. Se siguieron los siguientes pasos: Búsqueda de literatura relacionada; redacción del marco teórico-conceptual; definición del problema, establecimiento del diseño, elaboración del programa de intervención Mindfulness-ProAp, selección de cuestionarios y de participantes.

Fase de trabajo de campo: Información a los participantes del proceso de intervención; compromiso de asistencia; autorización de tratamiento de datos y seguimiento semanal; sesiones presenciales: cumplimentado de los tests, inventarios y autoinformes; entrenamiento específico y puesta en común experiencial.

En esta fase se aplicó el programa de entrenamiento Mindfulness-ProAp distribuido en cuatro sesiones presenciales con estructura similar: se comenzaba con la cumplimentación de las pruebas programadas para la sesión, se 
procedía con la puesta en común de los participantes sobre las prácticas realizadas en casa, se continuaba con la explicación y profundización de los bloques de los nuevos contenidos, alternando con las prácticas correspondientes, y se concluía la sesión con la solución de dudas o preguntas.

Las nuevas prácticas realizadas en la sesión presencial debían practicarse de forma individual en casa a lo largo de la semana, con la frecuencia e intensidad establecidas, con el fin de interiorizar las nuevas habilidades en el entrenamiento de la capacidad del desarrollo de la atención plena. Posteriormente se compartía en la siguiente sesión la experiencia vivida con su práctica, se exponía de forma oral todas las reflexiones personales derivadas de la práctica y las percepciones sentidas de la introducción de esta nueva práctica a sus vidas cotidianas. Estas aportaciones orales eran recogidas literalmente para realizar posteriormente el análisis cualitativo de la investigación.

Fase de análisis / resultados: Para la investigación cuantitativa se utilizó el programa estadístico SPSS versión 23 en castellano. Para la investigación cualitativa: transcripción de la puesta en común, establecimiento de categorías, definición de los códigos y glosarios; resultados obtenidos y elaboración de conclusiones.

\section{RESULTADOS Y DISCUSIÓN}

En las siguientes tablas se recogen los principales resultados obtenidos en las distintas pruebas aplicadas con la finalidad de conseguir los objetivos previstos. Primero se realiza el planteamiento de los resultados de análisis cuantitativos referidos a la normalidad de la muestra, a la media de atención plena y a la correlación entre las variables de atención plena y de inteligencia emocional. A continuación se exponen los resultados de los análisis de tipo cualitativo.

Tabla 1. Estadísticos descriptivos: Datos Inteligencia Emocional. (TMMS-24).

\begin{tabular}{lccccc}
\hline & N & Mínimo & $\begin{array}{c}\text { Máxim } \\
\text { o }\end{array}$ & Media & $\begin{array}{l}\text { Desviación } \\
\text { estándar }\end{array}$ \\
\hline IE Percepción & 111 & 13,00 & 40,00 & $\begin{array}{l}27,270 \\
3\end{array}$ & 6,19669 \\
\hline $\begin{array}{l}\text { IE } \\
\text { Comprensión }\end{array}$ & 111 & 12,00 & 40,00 & $\begin{array}{l}25,468 \\
5\end{array}$ & 5,70935 \\
\hline IE Regulación & 110 & 12,00 & 40,00 & $\begin{array}{l}27,100 \\
0\end{array}$ & 6,55667 \\
\hline $\begin{array}{l}\text { N válido (por } \\
\text { lista) }\end{array}$ & 110 & & & & \\
\hline
\end{tabular}

En la Tabla 1 se reflejan los datos obtenidos de la valoración de Inteligencia Emocional que confirman que la muestra se encuentra en un rango de normalidad. Las dimensiones valoradas son: Percepción ( $M=30$ y la Dt= 5), Claridad ( $M=30$ y la $\mathrm{Dt}=4$ ) y Regulación ( $\mathrm{M}=30$ y la $\mathrm{Dt}=4)$.

Estos datos nos confirman que los participantes en la investigación son sujetos que no presentan patologías ni alteraciones conductuales ni emocionales, por lo que nos permiten realizar la investigación desde la perspectiva de la psicología positiva.

Tabla 2. Análisis T-Test de Atención Plena APP / APF. Diferencias emparejadas.

\begin{tabular}{|c|c|c|c|c|c|c|c|c|c|}
\hline & & \multirow{3}{*}{$\begin{array}{c}\text { Med } \\
\text { ia }\end{array}$} & \multicolumn{4}{|c|}{ Diferencias emparejadas } & \multirow[b]{3}{*}{$\mathrm{t}$} & \multirow{3}{*}{\multicolumn{2}{|c|}{$\begin{array}{cc} & \text { Sig. } \\
& \text { (bilateral } \\
\text { gl } & \text { ) }\end{array}$}} \\
\hline & & & \multirow{2}{*}{$\begin{array}{l}\text { Desviaci } \\
\text { ón } \\
\text { estándar }\end{array}$} & \multirow{2}{*}{$\begin{array}{l}\text { Media de } \\
\text { error } \\
\text { estándar }\end{array}$} & \multicolumn{2}{|c|}{$\begin{array}{c}95 \% \text { de intervalo } \\
\text { de confianza de la } \\
\text { diferencia }\end{array}$} & & & \\
\hline & & & & & Inferior & Superior & & & \\
\hline Par & APPTO & & & & & & & & \\
\hline 1 & $\begin{array}{c}\text { TAL } \\
\text { Apftotal }\end{array}$ & ,486 & 12,502 & 1,198 & $-1,887$ & 2,860 & ,406 & 108 & ,686 \\
\hline
\end{tabular}


Al analizar los datos que nos aporta la prueba de diferencias de media entre las puntuaciones de atención plena antes y después de la intervención (tabla 2), se comprueba que estas diferencias no son estadísticamente significativas (T 2,108=.406, ns.).

Tabla 3. Correlaciones entre las medidas de atención plena y las variables de inteligencia emocional

\begin{tabular}{|c|c|c|c|c|c|c|c|}
\hline & & $\begin{array}{l}\text { APP } \\
\text { TOT } \\
\text { AL }\end{array}$ & $\begin{array}{l}\text { Apf } \\
\text { total }\end{array}$ & $\begin{array}{l}\text { Inc. } \\
\text { Ate } \\
\text { n. }\end{array}$ & $\begin{array}{l}\text { IE } \\
\text { Perc } \\
\text { ep. }\end{array}$ & $\begin{array}{l}\text { IE } \\
\text { Co } \\
\text { mp. }\end{array}$ & $\begin{array}{l}\text { IE } \\
\text { Reg } \\
\text { ul. }\end{array}$ \\
\hline \multirow[t]{3}{*}{$\begin{array}{l}\text { APP } \\
\text { TOTAL }\end{array}$} & $\begin{array}{l}\text { Corr. de } \\
\text { Pearson }\end{array}$ & 1 & & & & & \\
\hline & $\begin{array}{l}\text { Sig. } \\
\text { (bilateral) }\end{array}$ & & & & & & \\
\hline & $\mathrm{N}$ & 110 & & & & & \\
\hline \multirow[t]{3}{*}{ Apf total } & $\begin{array}{l}\text { Corr. de } \\
\text { Pearson }\end{array}$ & $\begin{array}{c}, 698^{*} \\
*\end{array}$ & 1 & & & & \\
\hline & $\begin{array}{l}\text { Sig. } \\
\text { (bilateral) }\end{array}$ &, 000 & & & & & \\
\hline & $\mathrm{N}$ & 109 & 111 & & & & \\
\hline \multirow{3}{*}{$\begin{array}{l}\text { Increme } \\
\text { nto } \\
\text { Atenció } \\
\mathrm{n}\end{array}$} & $\begin{array}{l}\text { Corr. de } \\
\text { Pearson }\end{array}$ & $\begin{array}{c}- \\
, 303^{*} \\
*\end{array}$ & $\begin{array}{l}, 471 \\
* *\end{array}$ & 1 & & & \\
\hline & $\begin{array}{l}\text { Sig. } \\
\text { (bilateral) }\end{array}$ &, 001 & ,000 & & & & \\
\hline & $\mathrm{N}$ & 109 & 109 & 109 & & & \\
\hline \multirow[t]{3}{*}{$\begin{array}{l}\text { IE } \\
\text { Percepci } \\
\text { ón }\end{array}$} & $\begin{array}{l}\text { Corr. de } \\
\text { Pearson }\end{array}$ &,- 164 & $\begin{array}{l}- \\
, 229 \\
*\end{array}$ & , 089 & 1 & & \\
\hline & $\begin{array}{l}\text { Sig. } \\
\text { (bilateral) }\end{array}$ & ,090 & ,016 &, 364 & & & \\
\hline & $\mathrm{N}$ & 108 & 109 & 107 & 111 & & \\
\hline \multirow{3}{*}{$\begin{array}{l}\text { IE } \\
\text { Compre } \\
\text { nsión }\end{array}$} & $\begin{array}{l}\text { Corr. de } \\
\text { Pearson }\end{array}$ & ,080 & ,168 &, 052 & $\begin{array}{l}, 328 \\
* *\end{array}$ & 1 & \\
\hline & $\begin{array}{l}\text { Sig. } \\
\text { (bilateral) }\end{array}$ & ,410 & ,081 &, 595 &, 000 & & \\
\hline & $\mathrm{N}$ & 108 & 109 & 107 & 111 & 111 & \\
\hline \multirow{3}{*}{$\begin{array}{l}\text { IE } \\
\text { Regulaci } \\
\text { ón }\end{array}$} & $\begin{array}{l}\text { Corr. de } \\
\text { Pearson }\end{array}$ &, $229 *$ & $\begin{array}{l}, 275 \\
* *\end{array}$ &, 105 &, 032 & $\begin{array}{l}, 221 \\
*\end{array}$ & 1 \\
\hline & $\begin{array}{l}\text { Sig. } \\
\text { (bilateral) }\end{array}$ &, 017 & ,004 & ,284 &, 744 &, 020 & \\
\hline & $\mathrm{N}$ & 107 & 108 & 106 & 110 & 110 & 110 \\
\hline
\end{tabular}


Estos datos nos confirman que antes del programa de intervención tan solo la variable de regulación emocional correlaciona significativamente con la mayor puntuación de atención plena. Además, nos indican que después del seguimiento del programa los sujetos que presentan mayor atención plena obtienen mejores puntuaciones en regulación emocional y comprensión emocional.

Esto nos hace inferir que el programa Mindfulness-ProAp no es útil para mejorar la atención plena, sino que, gracias a la comprensión emocional como variable significativa, conduce a una mejora de la toma de conciencia en atención plena. Este resultado es muy relevante y le da un alto valor a nuestro programa de entrenamiento porque con su aplicación se consigue que mindfulness no sea un solo una herramienta de práctica funcional, mecánica y física sino que, además, se transforme en un elemento cognitivo de autorregulación consciente.

\section{INVESTIGACIÓN CUALITATIVA}

Además de la investigación cuantitativa se llevó a cabo una investigación cualitativa mediante sesiones de focus group, en las que, en la puesta en común con el elemento diana de análisis experiencial, se recogían las valoraciones y la importancia que los/as participantes, como informantes clave por su experiencia obtenida durante el entrenamiento en mindfulness, atribuían a la toma de conciencia plena y a las habilidades y competencias emocionales. Optamos por esta opción porque nos parece la más apropiada para alcanzar un conocimiento exhaustivo de la experiencia, pues mediante la narrativa ofrece la posibilidad de acceder a información difícilmente cuantificable.

El análisis e interpretación de las aportaciones realizadas por los participantes en el programa, así como la codificación temática y la categorización nos ha permitido establecer 8 categorías y 18 subcategorías que se pueden incardinar entre las variables de inteligencia emocional como elementos que subyacen en el mayor autoconocimiento y en la gestión y afrontamiento de dificultades personales y en las relaciones sociales (Tabla 4).

Tabla 4. Resultados de los análisis de la valoración de programa Mindfulness: Categorías y verbatim. Categorías Verbatim

\begin{tabular}{ll}
\hline Valor de la toma de conciencia plena. "Considero que la atención plena contribuye a \\
un mejor autoconocimiento". \\
"La toma de conciencia me ha permitido ver las \\
situaciones estresantes no como bloqueadoras \\
sino como desafios". \\
"El programa me ha permitido pensar más en \\
las emociones que siento". \\
"Antes actuaba como un robot sin pensar en lo \\
que hacía". \\
\hline "He aprendido a nombrar e identificar mis \\
emociones. Ya no tengo miedo a lo que siento". \\
"Ahora entiendo mis emociones y por qué me \\
suceden" \\
\hline "Desde que participo en el programa intento ver \\
emocional \\
el lado positivo de las cosas. Antes me \\
angustiaba mucho". \\
"Desde que he asistido al programa mindfulness \\
sé manejar mejor mis emociones y afrontar sin \\
miedo los problemas"
\end{tabular}

International Journal of Developmental and Educational Psychology 


\begin{tabular}{ll}
\hline Afrontamiento de situaciones & "He conseguido un mayor autocontrol y esto ha \\
conseguido que me sienta mejor conmigo misma & y mejorado mis relaciones con los demás". \\
"Siento que me puedo sobreponer a cualquier \\
adversidad con menos dolor y sufrimiento". \\
Adaptabilidad \\
"Desde que estoy siguiendo el programa veo que \\
me adapto con más facilidad para respetar la \\
opinión de los demás. \\
"Antes tendia a rendirme a la primera, pero \\
ahora me siento con fuerzas para emprender \\
nuevos proyectos". \\
"Antes me machacaba mentalmente con mis \\
neuras. Ahora me siento más segura de mí misma \\
y menos ansiosa". \\
"Gracias a estas sesiones soy capaz de \\
valorarme más a mí misma. Creo que mi \\
autoestima está mejorando." \\
"Hasta ahora siempre he sido muy derrotista, \\
pero ya veo que puedo hacer las cosas sin tanto \\
miedo". \\
"Estas sesiones me permiten ser menos \\
desconfiado y manejar mejor mi estrés, lo que me \\
evita muchos conflictos". \\
\end{tabular}

\begin{tabular}{c}
\hline Gestión de conflictos interpersonales "Antes tomaba decisiones rápidas en la relación \\
que establecía con los demás, por lo que se me \\
generaban muchos conflictos. \\
"Sé decir las cosas con acierto y eso me evita \\
problemas con los demás". \\
"Me he dado cuenta que no siempre tengo que \\
ser el centro de atención. Eso me ha generado \\
muchos conflictos". \\
"Mis conflictos en casa han disminuido porque \\
soy capaz de comprender más a mi madre y a mi \\
hermana"
\end{tabular}

Los resultados de los análisis y la mayor cantidad de respuestas repetidas reflejan que la participación en el programa pautado y estructurado les ha permitido tener una percepción realista y positiva de la realidad sin exageraciones, un mayor control para actuar tanto ante las vivencias positivas como las negativas, mejorar las relaciones con los demás, una mayor capacidad de autoanálisis, adquirir más confianza en sí mismos y en los/as que le rodean y mayor capacidad de sobreponerse a las adversidades. Estos resultados nos resultaron reveladores por la importancia que atribuían a la atención plena y al autoconocimiento en el afrontamiento de conflictos tanto personales con en las relaciones con los demás.

Al igual que otras investigaciones (Shapiro, Schwart y Santerre 2002, Lyubomirsky, 2008, Siegel 2010, Kabat-Zinn 1990 y 2012) nuestro trabajo nos ha permitido demostrar que las personas que realizan la práctica del mindfulness reducen sus niveles de estrés, se sienten menos deprimidas y la ansiedad tiende a desaparecer. Como consecuencia de este bienestar aumenta el control de sus emociones y así disfrutan de mayor estabilidad emocional, crece la empatía y las relaciones con los demás mejoran notablemente. 
Así mismo, Figueroa, Contini, Lacunza, Levín y Estévez (2005) plantean que las personas que manifiestan mayor satisfacción con sus vidas, son los que desarrollan mejores habilidades, apreciación personal y un manejo adecuado de su entorno, y sobre todo hacen hincapié en el control de la tensión frente a situaciones de estrés, Si tenemos presente que uno de los beneficios de la práctica del mindfulness es el control del estrés comprobamos que los resultados obtenidos en nuestra investigación coinciden con esta aportación científica.

Por su parte, Goleman (1996), Redorta, Oriol y Bisquerra (2006) nos señalan la importancia de la regulación emocional para el manejo de las relaciones personales, aspecto que ha resultado relevante en nuestra investigación.

\section{CONCLUSIONES}

Una vez analizados los resultados y como conclusiones generales de esta investigación podemos afirmar que:

- Seguir un programa de entrenamiento pautado y estructurado de mindfulness mejora no solo la atención plena, sino que, además, facilita que se tome conciencia de atención plena.

- Existen variables emocionales, sobre todo de regulación emocional, que son responsables y que determinan el mayor beneficio de la competencia de atención plena

- La práctica del mindfulness, bajo pautas adecuadas, es útil para el afrontamiento de situaciones de estrés y para la gestión de conflictos intrapersonales e interpersonales.

- Una vez realizado el primer contacto con una metodología estructurada basada en mindfulness, las personas sobrevaloran su capacidad de atención plena y son más conscientes de sus carencias, ya que despierta una mayor conciencia y permite valorar de forma más objetiva su capacidad de atención plena como un aspecto de autorregulación consciente y no como una mera actividad física (respirar, relajarse,...). Es decir, el mindfulness no es un solo una herramienta de práctica funcional, mecánica y física sino que, además, se transforma en un elemento cognitivo de autorregulación consciente.

- La importancia que los participantes en la investigación atribuyen a las variables emocionales y a la atención plena en los conflictos personales e interaccionales nos permite reflexionar sobre la necesidad del conocimiento de estas variables por parte de los/as profesionales en la resolución de conflictos, para que en su actuación negociadora o mediadora faciliten a las personas en el proceso no solo resolver el conflicto de forma satisfactoria y de llegar a un acuerdo, sino la toma de conciencia de su transformación personal.

\section{REFERENCIAS BIBLIOGRFÁFICAS}

Fernández-Berrocal, P., Extremera, N. y Ramos, N. (2004). Validity and reliability of the Spanish modified versión of the Trail Meta - Mood Scale. Psychological Reports, 94, 751 - 755.

Figueroa, M. I., Contini, N., Lacunza, A. B., Levín, M., \& Suedan, A. E. (2005). Las estrategias de afrontamiento y su relación con el nivel de bienestar psicológico. Un estudio con adolescentes de nivel socioeconómico bajo de Tucumán (Argentina). Anales de Psicología / Annals of Psychology, 21(1), 66-72.

Gardner, H. (2001). La inteligencia reformulada: las inteligencias múltiples en el siglo XXI. Paidós.

Goleman, D. (1996). Inteligencia emocional. Kairós

Kabat-Zinn, J. (2003). Mindfulness-Based Stress Reduction (MBSR). Construtivism in de Human Sciences. Pág. 73-107.

Kabat-Zinn, J. (2012). Vivir con plenitud las crisis. Cómo utilizar la sabiduría del cuerpo y de la mente para afrontar el estrés, el dolor y la enfermedad. Kairós.

Hernández_Guanir, P. (2002). Los Moldes de la Mente. Más allá de la inteligencia emocional. Tafor Publicaciones.

Hernández_Guanir, P. (2010). Moldes. Test de estrategias cognitivas-emocionales. TEA Ediciones.

Inchausti, F., et al. (2013). Análisis Rasch de la versión española de la escala Mindful Attention Awareness Scale 
en una muestra clínica. Rev. Psiquiatr Salud Ment. http://dx.doi.org/10.1016/j.rpsm.2013.07.003

León, J., Fernández C., Grijalvo F., y Núñez, J. (2012)- Mindfulness awareness- attention scale: A five items versión. International Journal of Psychology, 47, 24-24.

Lyubomirsky, S. (2008). La ciencia de la felicidad: método probado para conseguir bienestar. Ediciones Urano.

Luján, I. (2015). Mediación y resolución de conflictos. Vicerrectorado de Profesorado y Planificación Académica Universidad de Las Palmas de Gran Canaria.

Luján, I. (2019) (Coord). Conflictos y mediación en contextos plurales de convivencia. Servicio de Publicaciones de la ULPGC.

Luján, I. y Díaz-Calcines, M. (2018). Importancia de la resiliencia en el proceso mediador. En F. Fariñas, M. Rosales, K Rolán, y M.J. Vázquez. Construcción de paz través de la mediación: Conocimientos y prácticas de una metodología. CUEMYC.

Martín, R., Luján, I. y Rodríguez-Mateo, H. (2015). Programa Mindfulness-ProAp en R. Martín, Perfil socioemocional de los que más se benefician de un programa Mindfulness. Tesis doctoral. Universidad de Las Palmas de Gran Canaria.

Redorta, J. (2019). Manual para la gestión y resolución de conflictos. Almuzara.

Redorta, J. (2021). Cómo abordar los conflictos estructurales. Almuzara

Redorta, J., Obiols, M y Bisquerra, R. (2006). Emoción y conflicto. Aprenda a manejar las emociones. Paidós.

Rodríguez-Trueba, C., Rodríguez-Mateo, H. y Luján, I. (2014). Validez predictiva del pensamiento constructivo en la eficacia negociadora. International Journal of Developmental and Psychology. Revista INFAD de Psicología, Vol 6, 1, 245-258.

Seligman, M. (2011). La vida que florece. Ediciones B.

Seligman, M. y Csikszentmihalyi, M. (2000). Positive Psychology. An Introduction. American Psychologist. January 2000 Vol. 55 №1, 5-14.

Shapiro, S., Schwartz, G. y Santene, C. (2002).Meditation and positive psychology. In C.R. Snyder y S.J. López (Ed.), Handbook of positive psychology (pp. 632-645). Oxford

Siegel, D. J. (2010). Cerebro y Mindfulness. Paidós.

Siegel, D. J. (2012). Mindfulness y Psicoterapia. Técnicas prácticas de atención plena para psicoterapeutas. Paidós.

Simón, V. (2015). La compasión: el corazón del mindfulness. Editorial Sello.

Vinyamata, E.(2019). Prólogo. En I. Luján, (Coord). Conflictos y mediación en contextos plurales de convivencia. Servicio de Publicaciones de la ULPGC. 